\title{
Substorm activity in Venus's magnetotail
}

\author{
M. Volwerk ${ }^{1}$, M. Delva ${ }^{1}$, Y. Futaana ${ }^{2}$, A. Retinò ${ }^{1}$, Z. Vörös ${ }^{3}$, T. L. Zhang ${ }^{1}$, W. Baumjohann ${ }^{1}$, and S. Barabash ${ }^{2}$ \\ ${ }^{1}$ Space Research Institute, Austrian Academy of Sciences, 8042 Graz, Austria \\ ${ }^{2}$ Swedish Institute of Space Physics, Kiruna, 98128, Sweden \\ ${ }^{3}$ Institute of Astro- and Particle Physics, University of Innsbruck, 6020 Innsbruck, Austria
}

Received: 24 September 2008 - Revised: 13 May 2009 - Accepted: 25 May 2009 - Published: 8 June 2009

\begin{abstract}
The magnetotail of the induced magnetosphere of Venus is investigated through the magnetic field and plasma data of Venus Express. A comparison is made between two neutral sheet crossings. One crossing shows the magnetic field is rather quiet and the plasma instrument indicates a change from energetic (few $100 \mathrm{eV}$ ) to low energy (few $10 \mathrm{eV}$ ) ions. The other crossing shows more dynamics in the magnetic field, including signatures that are interpreted as characteristic of a reconnection site, and the plasma instrument indicates ions that are energized to 1500 to $2000 \mathrm{eV}$, in the same magnetospheric region where in the first crossing only low energy ions showed up.
\end{abstract}

Keywords. Magnetospheric physics (Magnetotail; Planetary magnetospheres) - Space plasma physics (Magnetic reconnection)

\section{Introduction}

The field of physics of planetary magnetotails has expanded greatly with the various missions around the Earth and other planets. Many phenomena, like e.g. substorms, in which possible reconnection reconfigures the topology of the magnetic field in the tail, (for a review see, e.g., Baker et al., 1996) are not only taking place in the Earth's tail. Various observations have shown that a similar process may be taking place, not only at magnetized planets like Jupiter (Kronberg et al., 2005, 2008; Ge et al., 2007) and Saturn (Mitchell et al., 2005; Jackman et al., 2007; Russell et al., 2008) and a possible indication at Mercury (Fujimoto et al., 2007), but also at unmagnetized planets like Mars (Eastwood et al., 2008), at comets (Russell et al., 1986; Vourlidas et al., 2007) and Titan (Bertucci et al., 2008) where the solar wind magnetic

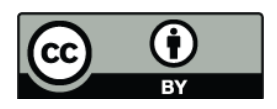

Correspondence to: $\mathrm{M}$. Volwerk (martin.volwerk@oeaw.ac.at) field drapes around the object, creating a so-called induced magnetosphere and tail. Recently, Fedorov et al. (2008) discussed the similarities between the magnetotails of Venus and Mars, using Mars Express and Venus Express plasma data.

However, reconnection is not the only process which can reconfigure the magnetotail. In addition, current disruption and the ballooning instability (for a discussion of the distinction between these three processes see e.g. Haerendel, 1992; Parker, 1996; Lui, 2000; Baumjohann et al., 2007) and associated processes before and after their onset in the Earth's magnetotail re-arrange the magnetic configuration of the tail. In this paper, however, we will concentrate on the reconnection process, for which we think the data show most evidence.

In this paper, we will investigate the crossing of the induced magnetotail of Venus by Venus Express (VEX). During the orbits in summer 2006, the spacecraft passed through the magnetotail from the ecliptic south to the north, heading towards periapsis above Venus's north pole. Venus does not have an intrinsic magnetic field, however, the magnetic field of the solar wind interacts with this planet to create an induced magnetosphere. Similar to the Earth, there is a bow shock, a magnetosheath and a magnetopause/ionopause (see, e.g., Zhang et al., 2007, 2008a,b, for recent VEX results).

The draping of the magnetic field creates a magnetotail on the downstream side of the planet (see e.g. Phillips and McComas, 1991). In the near-Venus magnetotail, i.e. at distances less than $\sim 4 R_{V}\left(R_{V}\right.$ is Venus's radius, $6051.8 \mathrm{~km}$ ), where VEX crosses through the tail, there is little information about the structure of the magnetic field. Murabashi et al. (1985) showed that there can be converging of the magnetic field in the near-tail, based on a study of PVO magnetic field data. Russell et al. (1981) found that the magnetotail has an enhanced magnetic field strength, compared with the magnetosheath, and that the direction of the field is basically aligned with the solar wind direction. The tail-lobe field

Published by Copernicus Publications on behalf of the European Geosciences Union. 
Table 1. Solar wind magnetic field. The value of the field is based on an average over 15 min of low-pass filtered data, 15 min before and after crossing the bow shock.

\begin{tabular}{l|rrrrr|rrrrr}
\hline & \multicolumn{7}{|c|}{ inbound } & \multicolumn{5}{c}{ outbound } \\
Event & $B_{x}$ & $B_{y}$ & $B_{z}$ & $B_{m}$ & $\theta_{\mathrm{P}}$ & $B_{x}$ & $B_{y}$ & $B_{z}$ & $B_{m}$ & $\theta_{\mathrm{P}}$ \\
$\mathrm{nT}$ & $\mathrm{nT}$ & $\mathrm{nT}$ & $\mathrm{nT}$ & $\mathrm{deg}$ & $\mathrm{nT}$ & $\mathrm{nT}$ & $\mathrm{nT}$ & $\mathrm{nT}$ & $\mathrm{deg}$ \\
\hline $1(15 \mathrm{Aug})$ & -7 & 7 & -8 & 13 & 123 & -9 & 13 & -12 & 19 & 117 \\
$2(22 \mathrm{Aug})$ & -2 & 7 & 5 & 9 & 103 & -5 & -4 & -5 & 8 & 128 \\
\hline
\end{tabular}

was found to be $\sim 83 \%$ stronger than in the magnetosheath. The authors also showed that the crossing from the magnetosheath to the tail lobe was characterized by a change in the wave spectrum. Indeed, Vörös et al. (2008a,b) found that the turbulence characteristics change when the spacecraft moves from the magnetosheath, to the (extended) wake region, into the planetary shadow.

In this paper we will discuss the data for two different magnetotail crossings, the first crossing shows some activity in the tail with VEX moving from the tail to the nearVenus magnetosphere, showing a significant jump in ion energy from high to low and a change of flow direction from tailward to azimuthal after crossing the neutral sheet; the second crossing also shows some magnetic activity in the tail, however it first shows an increase in ion energy after the crossing of the neutral sheet which could be interpreted as evidence for reconnection. We use data from both the magnetometer MAG (Zhang et al., 2006) and the plasma instrument ASPERA-4 (Barabash et al., 2007a). Unfortunately, we are not able to provide plasma moments in this paper, only spectra. Energy spectra are obtained every $24 \mathrm{~s}$ for one of 8 elevation angles of the detector and either summed over all 16 azimuthal channels for a summary view, or all azimuthal directions are viewed separately (both kinds of spectra will be shown in this paper).

Throughout the paper we use the Venus Solar Orbital (VSO) coordinate system, which is centred on Venus with the $\mathrm{X}$-axis is in the direction to the Sun, the Y-axis in the opposite direction of Venus's orbital motion and the Z-axis is perpendicular and positive towards the ecliptic north.

\section{The events}

\subsection{Event 1: 15 August 2006 - a quiet current sheet crossing}

On 15 August 2006, VEX crossed Venus's magnetotail during the inbound phase of the orbit. The magnetic field and plasma data, and the location of the spacecraft are shown Fig. 1. VEX enters the magnetotail from the ecliptic south, and first VEXMAG measures a negative (tailward) $B_{x}$. VEX passes through a rather active Southern Hemisphere, showing large excursions in the magnetic field, with
$B_{x}$ even slightly crossing into the Northern Hemisphere. At $\sim 01: 48$ UT, VEX crosses the neutral sheet $\left(B_{m} \approx 0\right)$ into the Northern Hemisphere, where positive $B_{x}$ is measured and a rather stable field exists.

The solar wind conditions during this event change slightly in the period before entering and after exiting the bow shock, see Table 1 . The magnetic field magnitude of the solar wind was above average at $B_{m} \approx 13 \mathrm{nT}$, where the average value $B_{m} \approx 8 \mathrm{nT}$ for solar minimum was found from VEX data. The previous day, 14 August, after 23:00 UT the solar wind had approximately double the average magnetic field strength, which will lead to a stronger field in the induced magnetosphere. The field reduced again to almost average before VEX entered through the bow shock at $\sim 00: 16$ UT. $B_{y}$ changed significantly in the last hours of 14 August, from negative to positive.

The magnetometer data in Fig. 1 (middle panels) show that during the neutral sheet (current sheet) crossing in the inter$\mathrm{val}, \sim 01: 47: 50-\sim 01: 48: 46 \mathrm{UT}$, the magnetic field is almost exclusively in the $X_{\mathrm{VSO}}$-direction and changes from $B_{x} \approx$ $-27 \mathrm{nT}$ to $B_{x} \approx 27 \mathrm{nT}$. Indeed, Minimum Variance Analysis (MVA, Sonnerup and Scheible, 1998) of the current sheet crossing reveals eigenvalues $[4,18,662]$ with eigenvectors $(0,0.3,0.9),(0,0.9,-0.3)$ and $(-1,0,0)$, indicating a well determined system and a current sheet normal, determined by the minimum variance direction, only slightly tilted from $Z_{\mathrm{VSO}}$.

The plasma data are shown in Fig. 1 top panels. There is a $\sim 3$ min cadence in the data, which is caused by the data sampling method as explained above in the introduction. It is seen that before the crossing of the neutral sheet the ions have maximum intensity at a few $100 \mathrm{eV}$, whereas after the crossing the maximum intensity is at a few $10 \mathrm{eV}$, indicating that the spacecraft has entered a different region in Venus's induced magnetosphere, e.g. a region of closed field lines around the planet. The draped magnetic field lines are moved along with the solar wind, also in the "outflow region" of the magnetosheath. The average solar wind velocity at solar minimum is $v_{\mathrm{sw}} \approx 410 \mathrm{~km} / \mathrm{s}$ (see e.g. Luhmann et al., 1997), which, for protons corresponds to an energy of $E=0.5 m_{\mathrm{p}} v_{\mathrm{sw}}^{2} \approx 870 \mathrm{eV}$, which is above the observed energy of the observed protons at $\sim 100 \mathrm{eV}$ (except for the first peak near 01:31 UT). We suggest, therefore, that the plasma 


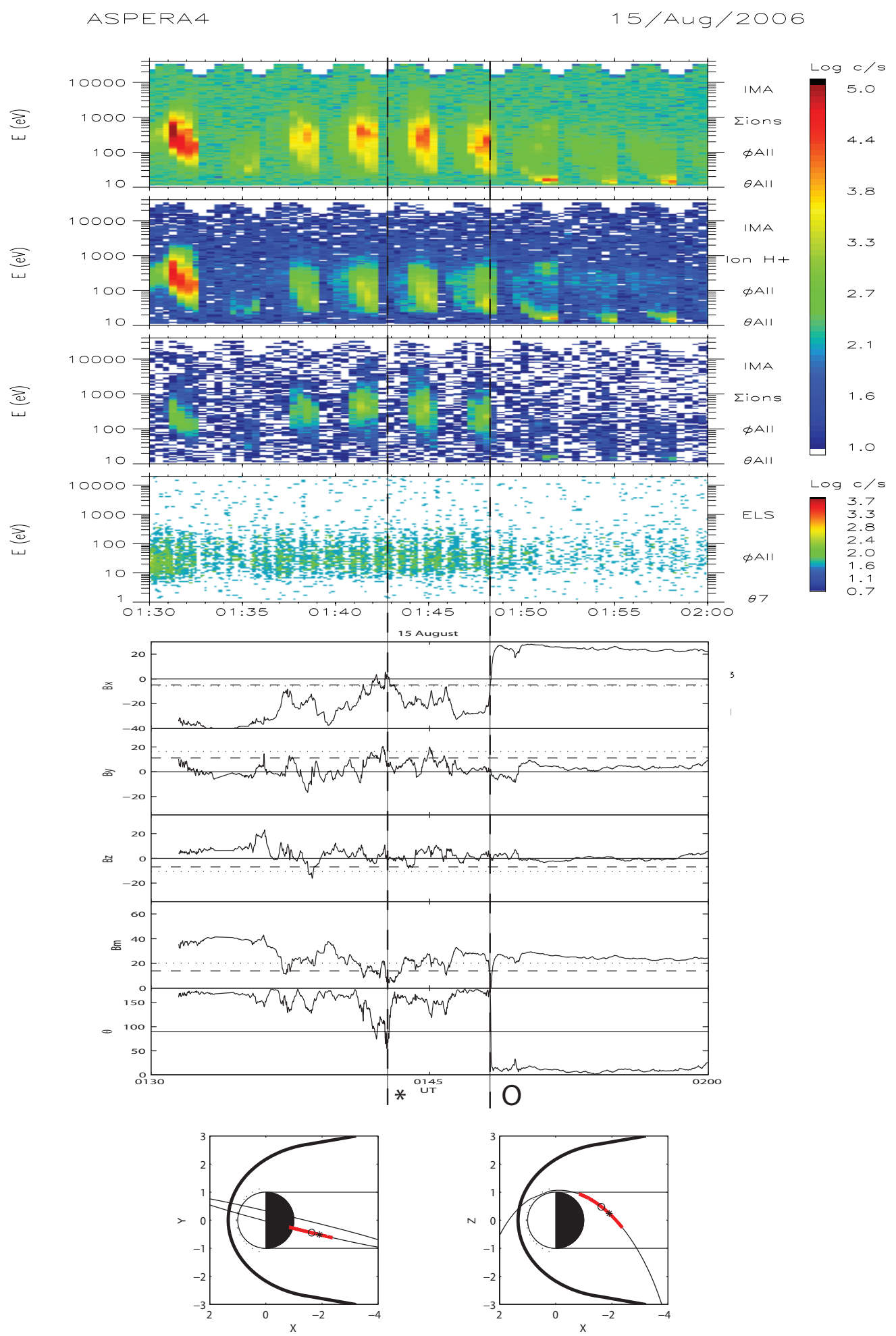

Fig. 1. Plasma and magnetic field data for 15 August 2006. Shown are the ASPERA data (total ions, $\mathrm{H}^{+}$, heavy ions, electrons) integrated over all azimuths, the VEXMAG magnetic field data $B_{x}, B_{y}, B_{z}, B_{m}$, and elevation angle $\theta$, and the orbit of VEX in the $X Y_{\mathrm{VSO}}$ and $X Z_{\mathrm{VSO}}$-plane, with the part of interest, for which the data are shown in the upper panels, colored red. The dashed vertical lines indicate specific times of interest and the asterisk and circle correspond to the same symbols in the bottom panels. 
Table 2. Viewing directions of ASPERA for the energized particles for 15 August 2006 for the three sectors $\theta$ showing largest count rate, the low-pass filtered magnetic field direction $\mathbf{B}$ and the angle $\Phi_{\theta, B}$ between viewing direction with maximum counts (bold) and magnetic field direction and the angle $\Phi_{\theta, s}$ between viewing direction with maximum count and solar direction (i.e. X-axis).

\begin{tabular}{lcccccc}
\hline UT & $\theta 1$ & $\theta 2$ & $\theta 3$ & $\mathbf{B}$ & $\Phi_{\theta, B}$ & $\Phi_{\theta, s}$ \\
\hline $01: 44: 44$ & $(\mathbf{0 . 8 8},-\mathbf{0 . 1 8},-\mathbf{- 0 . 4 5})$ & $(0.85,-0.46,-0.25)$ & $(0.69,-0.71,-0.08)$ & $(-19,8,5)$ & $164^{\circ}$ & $28^{\circ}$ \\
$01: 45: 07$ & $(\mathbf{0 . 7 7},-\mathbf{0 . 3 0},-\mathbf{0 . 5 7})$ & $(0.75,-0.54,-0.39)$ & $(0.61,-0.76,-0.23)$ & $(-17,9,7)$ & $165^{\circ}$ & $40^{\circ}$ \\
$01: 45: 33$ & $(\mathbf{0 . 7 7 , 0 . 4 7 , 0 . 4 3 )}$ & $(0.75,0.24,0.62)$ & $(0.62,0.02,0.79)$ & $(-16,9,6)$ & $91^{\circ}$ & $40^{\circ}$ \\
\hline UT & $\theta 1$ & $\theta 2$ & $\theta 3$ & $\mathbf{B}$ & $\Phi_{\theta, B}$ & $\Phi_{\theta, s}$ \\
\hline $01: 47: 57$ & $(0.87,-0.23,-0.44)$ & $(0.86,-0.47,-0.19)$ & $(\mathbf{0 . 7 3},-\mathbf{0 . 6 8 , 0 . 0 4})$ & $(-15,1,1)$ & $140^{\circ}$ & $43^{\circ}$ \\
$01: 48: 20$ & $(0.76,-0.37,-0.53)$ & $(0.76,-0.57,-0.31)$ & $(\mathbf{0 . 6 4},-\mathbf{0 . 7 6},-\mathbf{0 . 1 0})$ & $(1,-1,3)$ & $71^{\circ}$ & $50^{\circ}$ \\
$01: 48: 45$ & $(0.76,0.59,0.29)$ & $(0.76,0.39,0.52)$ & $(\mathbf{0 . 6 5}, \mathbf{0 . 2 1}, \mathbf{0 . 7 3})$ & $(18,-4,3)$ & $47^{\circ}$ & $50^{\circ}$ \\
\hline UT & $\theta 4$ & $\theta 5$ & $\theta 6$ & $\mathbf{B}$ & $\Phi_{\theta, B}$ & $\Phi_{\theta, s}$ \\
\hline $01: 51: 09$ & $(0.55,-0.77,0.32)$ & $(\mathbf{0 . 2 4},-\mathbf{0 . 8 6 , 0 . 4 5})$ & $(-0.10,-0.87,0.48)$ & $(26,6,-3)$ & $90^{\circ}$ & $76^{\circ}$ \\
$01: 51: 32$ & $(0.49,-0.86,0.17)$ & $(\mathbf{0 . 2 2},-\mathbf{0 . 9 3}, \mathbf{0 . 2 9})$ & $(-0.08,-0.95,0.32)$ & $(26,5,-3)$ & $90^{\circ}$ & $77^{\circ}$ \\
$01: 51: 57$ & $(0.49,0.24,0.84)$ & $\mathbf{( 0 . 2 3 , 0 . 1 7 , 0 . 9 6})$ & $(-0.07,0.15,0.98)$ & $(26,3,-2)$ & $80^{\circ}$ & $77^{\circ}$ \\
\hline
\end{tabular}

instrument is observing slowed down solar wind ions before the crossing of the neutral sheet. Indeed, the maxima in the ion counts appear in the azimuthal channels of ASPERA that are viewing in the sunward direction, as shown in Table 2. These signatures are in agreement with the ion outflow measured by Barabash et al. (2007b).

For three consecutive maxima in the count rate by ASPERA (at $\sim 01: 45, \sim 01: 48$, and $\sim 01: 51$ UT), we have studied the relationship between viewing direction, solar direction and magnetic field direction. The first maximum occurs when VEX is in the tail near the neutral sheet. It is found that the viewing direction for maximum counts is at an angle of $\leq 40^{\circ}$ from the solar direction, but does not seem to have a specifically defined angle with the magnetic field.

As the spacecraft crosses the current sheet during the second maximum at 01:48 UT, the situation is not much different. However, when ASPERA starts measuring the lowenergy ions at 01:51 UT, the direction has turned to almost perpendicular to the solar direction and perpendicular to the magnetic field. The protons show an energy signature of $\sim 20 \mathrm{eV}$, which would translate to a flow velocity of $\sim 10 \mathrm{~km} / \mathrm{s}$, indicating a slow flow, possibly around the planet, closer to Venus. This would fit the model by Brace and Kliore (1991) with slow flow in an extended ionosphere on the night side of Venus.

Although the purpose of the paper is to investigate the difference between a current sheet crossing without and with apparent reconnection, we also have to look at what happened before the current sheet crossing, during the time interval 01:35-01:45 UT, where the magnetometer measures an approaching of the neutral sheet and even a slight crossing of it at the time of the first vertical dashed line in Fig. 1 marked with an asterisk at the bottom. There are two "dips" into the centre of the magnetotail (or into the central current sheet) for which $B_{x}$ recovers (first dip) to almost the starting value and recovers to approximately half the starting value (second dip). Just before the current sheet crossing discussed above, the magnetic field almost recovers to its value at the start of this event. We will go through this interval dip-by-dip.

For the first dip at $\sim 01: 37$ UT the signature in the magnetic field shows an increase in $B_{z}$ and $B_{y}$ and a slight decrease in $B_{x}$, which can be interpreted as a dipolarization of the field. At $\sim 01: 40$ UT the $B_{x}$ has returned to almost its old value, as have the other components. Concentrating on the region between the two red lines in Fig. 2 we observe a bipolar structure in $B_{y}$, a monotonic decrease in $B_{z}$ and an increase and decrease in $B_{x}$, with the maximum negative value in $B_{x}$ occurring as the other two components cross zero. This gives the impression of a flux rope with a guide field (see, e.g., Henderson et al., 2006) in the X-direction, which is rather unusual in the Earth's magnetotail. Because of the fluctuations on the signal, minimum variance analysis does not produce a very well defined base (eigen values 20,30 and 80 ), however it does show an increase and decrease in field strength in the minimum variance direction $(X)$, a bipolar signature in the intermediate variance direction $(Z)$ and a rotation in the maximum variance direction $(Y)$. With the bipolar structure in $B_{y}$ this means that the flux rope is moving in the Zdirection. Unfortunately, with just this one crossing, we cannot deduce whether this flux tube originates from the solar wind or from the tail magnetic field. The plasma data for this interval show proton peak intensities near $300 \mathrm{eV}$ (corresponding to a velocity of $v_{\mathrm{p}} \approx 240 \mathrm{~km} / \mathrm{s}$ compared with a solar wind velocity of $\sim 410 \mathrm{~km} / \mathrm{s}$ ) similar to the next three signals in the ASPERA data. The maximum count rate is observed in $\theta 1$ from the $(0.96,0.05,-0.29)$ viewing direction, 

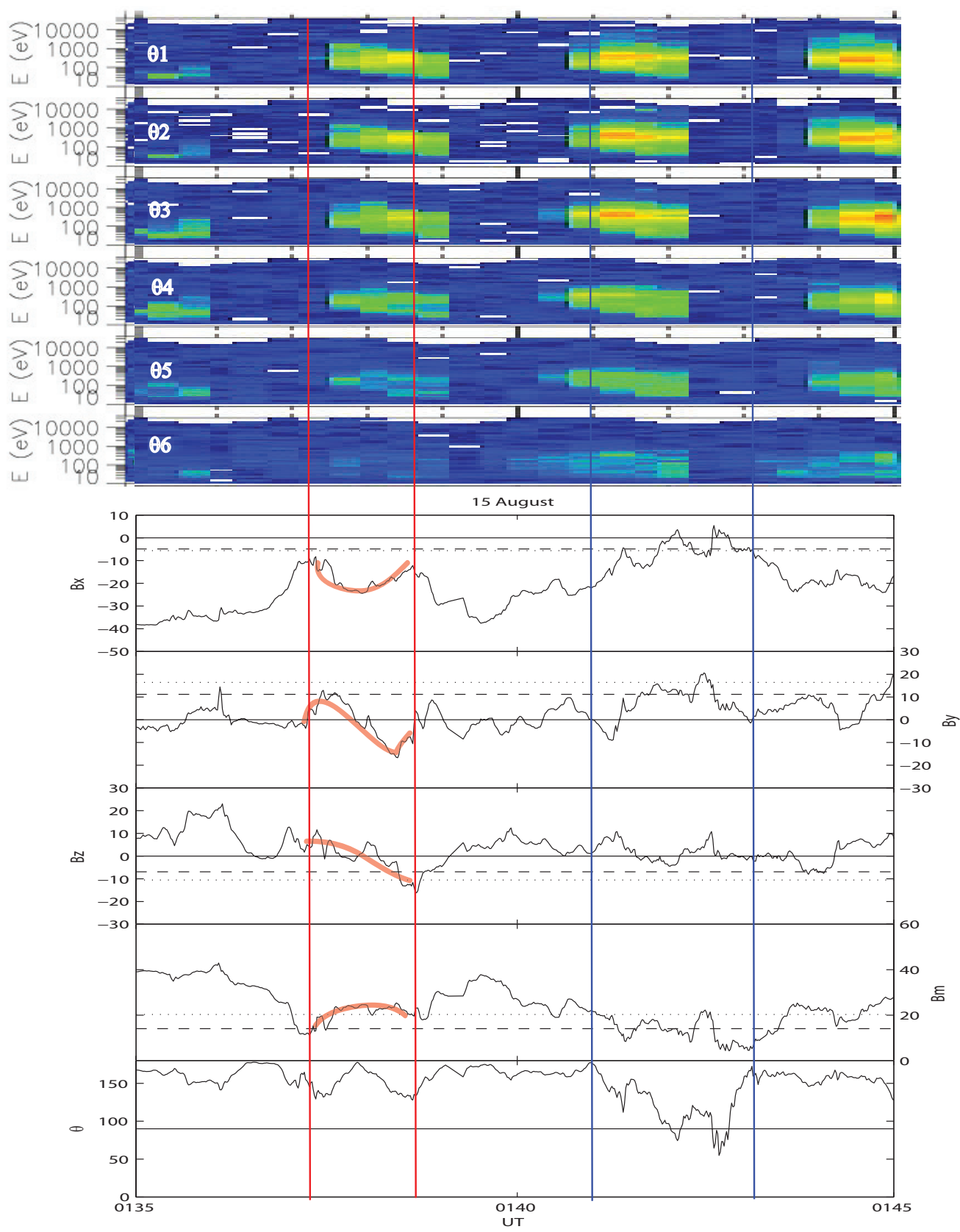

Fig. 2. Top: Six azimuthal viewing directions $(\theta 1$ to $\theta 6)$ from ASPERA, which show the maximum count rate. Bottom: A zoom in of the magnetometer and plasma data for 15 August 2006. Between the two red lines, magnetic signatures implicate a magnetic flux rope in Venus's magnetotail. Between the two blue lines, VEX enters into the central current sheet and observes low-frequency oscillations of the field.

which is almost similar to the minimum variance direction of the flux tube $(0.94,0.14,-0.29)$, indicating that the ions are flowing along the guide field of the flux tube.

The second dip interval, between the two blue lines in Fig. 2 is different: $B_{x}$ decreases in strength until it crosses the "neutral sheet" and enters into the other tail hemisphere, with low frequency oscillations of the field (large oscillations at $\sim 40 \mathrm{~s}$ and small amplitude waves at $\sim 5 \mathrm{~s}$ ) superimposed onto the field in the separate components and in the field magnitude $B_{m}$. These look like compressional waves near the neutral sheet, as observed by Volwerk et al. (2003, 2004) in the Earth's magnetotail. Most likely, Venus's magnetotail is flapping, moving the spacecraft towards the neutral sheet and then back out again, which could also explain the motion of the flux tube in the Z-direction mentioned above. 


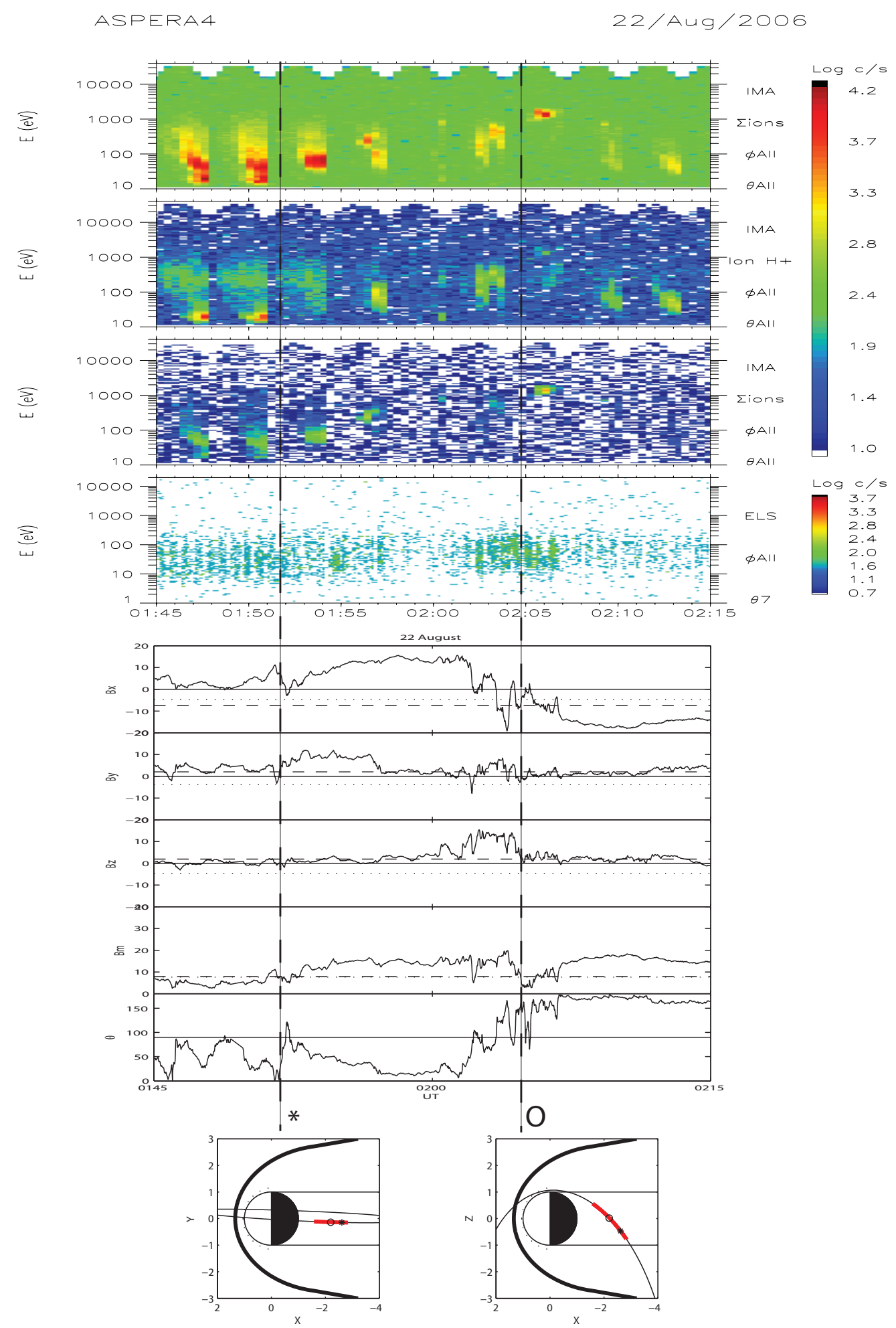

Fig. 3. Plasma and magnetic field data for 22 August 2006. Shown are the ASPERA data (total ions, $\mathrm{H}^{+}$, heavy ions, electrons) integrated over all azimuths, the VEXMAG magnetic field data $B_{x}, B_{y}, B_{z}, B_{m}$, and elevation angle $\theta$, and the orbit of VEX in the $X Y_{\mathrm{VSO}}$ and $X Z_{\mathrm{VSO}}$-plane, with the part of interest colored red. The dashed vertical lines indicate specific times of interest and the asterisk and circle correspond to the same symbols in the bottom panels. 


\subsection{Event 2: 22 August 2006 - a substorm event}

One of the characteristic (chain of) processes in a magnetotail is a substorm, which reconfigures the magnetic topology of the tail. One of the primary signs of a substorm is a dipolarization of the magnetic field, i.e., a turning of the stretched tail like magnetic field into a more dipolar like configuration (see, e.g., Baumjohann et al., 1999). These substorms are also characterized by plasma flows and particle energization (see, e.g., Imada et al., 2005, 2007).

On 22 August 2006, VEX crossed Venus's magnetotail during the inbound part of the orbit. The magnetic field and plasma data, and the location of the spacecraft are shown Fig. 3. VEX entered the tail from the south, and first VEXMAG measures a positive $B_{x}$, this in contrast to Event 1 and indicates that the solar wind magnetic field direction now is pointed southward.

At $\sim 02: 02$ UT there is an dipolarization of the magnetic field, indicated by an increase of $B_{z}$ combined with a decrease of $B_{x}$. However, there is also a signature in $B_{y}$ related to this dipolarization, and thus it is possible that the induced magnetotail does not have its current sheet in the XY-plane. Therefore, we transform the data in the interval 02:03-02:09 UT into a minimum variance coordinate system (Sonnerup and Scheible, 1998). The eigenvalues are [3, 18, $121]$ with eigenvectors $(-0.03,-0.93,0.36),(0.17,-0.36$, $-0.92)$ and $(0.98,0.03,0.17)$ with respect to the VSO coordinate system. The ratio of the minimum and intermediate eigenvalue is small, however, using nested MVA analyses (see, e.g., Volwerk, 2006) and shifting the interval slightly shows a consistent direction of the eigenvectors. The minimum variance direction shows that the normal of the current sheet, described by the eigen vector belonging to the minimum eigen value, is basically along the Y-direction. The result of the transformation into the minimum variance coordinate system can be seen in Fig. 4, where the magnetic field is displayed in $B_{\mathrm{MIN}}, B_{\mathrm{MED}}$ and $B_{\mathrm{MAX}}$ components and the total magnetic field $B_{m}$.

A dipolarization of the magnetic field now shows itself as a rotation from the maximum into the minimum variance direction, marked by the first red vertical line in Fig. 4. This is what is observed just after 02:02 UT. As mentioned, these dipolarizations are usually accompanied by plasma flows. In Fig. 4, the ASPERA data are also shown; $\theta 1$ through $\theta 6$ of the 16 different viewing directions of the detector, which have maximum count rates. For these six viewing directions the time-energy spectra of the detector counts are shown. The maximum count rate is in $\theta_{3}$ at $\sim 200 \mathrm{eV}$, which has a viewing direction (at this time) of $(0.69,0.71,0.16)$.

The intermediate direction, basically along the $\mathrm{Z}$ direction, shows that the field is varying around $B_{\mathrm{MED}} \approx$ $-5 \mathrm{nT}$ during the interval of 02:00-02:09 UT. This indicates a shear magnetic field (or guide field) along the cross tail current, which is not unusual in e.g. the Earth's magnetotail (see, e.g., Petrukovich et al., 2005).

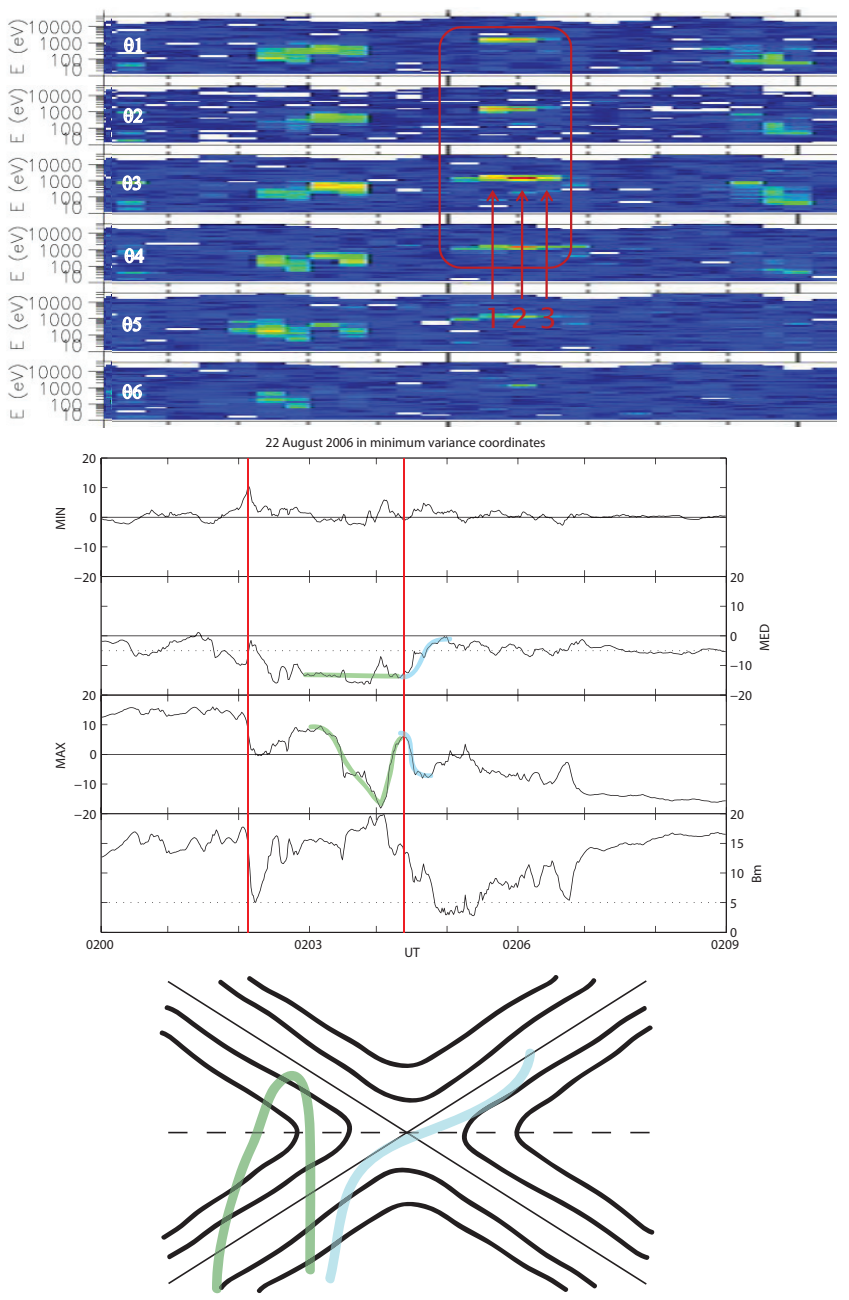

Fig. 4. Top: 6 viewing directions of ASPERA ( $\theta 1$ to $\theta 6)$ which show the maximum count rate. The red box and arrows indicate the three data points of energized ions, discussed in the text and in Table 3. Middle: The magnetic field data for 22 August 2006 in minimum variance coordinates for the interval 02:00-02:09 UT. Bottom: A schematic view of the motion of the spacecraft through the reconnection region. The green and blue line correspond to the green and blue lines in the top panels.

After the initial dipolarization there is an increase in $B_{\mathrm{MED}}$ from $\sim-5$ to $\sim-12 \mathrm{nT}$, and VEX starts crossing the current sheet near $\sim 02: 03$ UT (coloured in green in Fig. 4). The increased $B_{\mathrm{MED}}$ can be interpreted as Hall magnetic field created by reconnection in the tail (see e.g., Sonnerup, 1979; Runov et al., 2003) (see also bottom panel of Fig. 4). As VEX crosses $B_{\mathrm{MAX}} \approx 0 \mathrm{nT}$, there are high count rates in the ASPERA data with maximum at $\theta 3$, whose viewing directions of ASPERA are listed in Table 3. The table shows that the ions are moving tailward, at an angle of $65-70^{\circ}$ with respect to the magnetic field. This is what would be expected for the outflow region of a reconnection site. Indeed, the ions have a higher energy than those measured before the event at e.g. $\sim 01: 45-01: 55$ UT. 
Table 3. Viewing directions of ASPERA for the energized particles for 22 August 2006 for the three sectors $\theta$ showing largest count rate, the low-pass filtered magnetic field direction $\mathbf{B}$ and the angle $\Phi_{\theta, B}$ between viewing direction with maximum counts (bold) and magnetic field direction and the angle $\Phi_{\theta, s}$ between viewing direction with maximum count and solar direction (i.e. X-axis). The peak in energized ions is partly perpendicular for the first time, whereas mainly field aligned during the next two times.

\begin{tabular}{l|cccccc}
\hline UT & $\theta 1$ & $\theta 2$ & $\theta 3$ & $\mathbf{B}$ & $\Phi_{\theta, B}$ & $\Phi_{\theta, s}$ \\
\hline $02: 03: 06$ & $(0.64,-0.64,0.42)$ & $(0.83,-0.35,0.44)$ & $(\mathbf{0 . 8 8},-\mathbf{0 . 0 1 , 0 . 4 7})$ & $(-1,5,13)$ & $65^{\circ}$ & $28^{\circ}$ \\
$02: 03: 29$ & $(0.57,-0.58,0.58)$ & $(0.72,-0.33,0.61)$ & $(\mathbf{0 . 7 7}-\mathbf{0 . 0 3 0 . 6 3})$ & $(-4,5,13)$ & $70^{\circ}$ & $40^{\circ}$ \\
\hline $1-02: 05: 54$ & $(0.71,-0.67,0.21)$ & $(0.90,-0.36,0.25)$ & $\mathbf{( 0 . 9 6 , 0 . 0 1 , 0 . 2 9})$ & $(-7,1,3)$ & $139^{\circ}$ & $16^{\circ}$ \\
$2-02: 06: 18$ & $(0.66,-0.65,0.39)$ & $(0.83,-0.35,0.43)$ & $(\mathbf{0 . 8 8},-\mathbf{0 . 0 2}, \mathbf{0 . 4 7})$ & $(-4,1,1)$ & $154^{\circ}$ & $20^{\circ}$ \\
$3-02: 06: 41$ & $(0.57,-0.60,0.56)$ & $(0.73,-0.34,0.59)$ & $(\mathbf{0 . 7 7},-\mathbf{0 . 0 5}, \mathbf{0 . 6 3})$ & $(-4,1,1)$ & $154^{\circ}$ & $20^{\circ}$ \\
\hline
\end{tabular}

As the spacecraft crosses the current sheet again at $\sim 02: 04$ UT there is no signature of increased ion count rates. At the start of this crossing there is a decrease of $B_{\mathrm{MED}}$, which returns again to its previous value.

Just after 02:04 UT, at the second vertical red line in Fig. 4 there is again a crossing of the current sheet (coloured blue), however, in this case there is a simultaneous turning of the

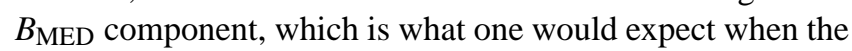
spacecraft crosses the reconnection site from one side to the other. Again, during this interval there are no increased ion count rates in the ASPERA data.

First one minute later, there are increased count rates at rather elevated energies of the ions in the red box with arrows in Fig. 4. At this time the $B_{\mathrm{MED}}$ component only varies slightly around the average value of $-5 \mathrm{nT}$ (the value of the assumed shear field in the tail) and thus a Hall magnetic field is not present anymore, indicating that reconnection either has stopped or the spacecraft has moved too far from the site. The energized ions that are observed are, again, moving tailward (see Table 3). The energy of the ions is much higher than before the dipolarization, now at 1500 to $2000 \mathrm{eV}$ and they move at a small angle with the magnetic field.

The question to answer now is where these energized ions come from. If there is reconnection of draped field, then the process creates closed loops around the planet. VEX is at $X_{\mathrm{VSO}} \approx-2 R_{\mathrm{V}}$, and approximating the closed field line by a circle of $\sim 2.5 R_{\mathrm{V}}$ diameter, one finds that the ions would take approximately $90 \mathrm{~s}$ to go around (at $1500 \mathrm{eV}$ the proton velocity is $530 \mathrm{~km} / \mathrm{s}$ ). Another process sending the ions back tailward, after being accelerated planetward is through magnetic mirroring, as the magnetic field strength will increase when the particles get closer to Venus and try to move around it (see, e.g., Zhang et al., 2008a). The latter option is preferable considering the rather large angle between the ions and the magnetic field.

After this, the plasma energy spectra show low energy particles again as VEX nears periapsis, similar to event 1 .

\section{Conclusions}

In this paper we studied two Venus magnetotail crossings by the Venus Express spacecraft. Both crossings showed activity in Venus's magnetotail, but very different from each other. The main purpose of this paper was to search for evidence of possible substorm or reconnection activity in Venus's induced magnetotail. We have shown that in a rather fast crossing from the southern to the Northern Hemisphere, in Event 1, the plasma population changed from moderately energetic (few $100 \mathrm{eV}$ ) to very low energy ions (few $10 \mathrm{eV}$ ). On the other hand, in Event 2, there were magnetic signatures which indicate that VEX was near a reconnection site and close by energized ions were measured by the plasma instrument. Although the energized ions moved in the tailward direction, as opposed to the expected planetward direction, we still interpret this as a reconnection event. The ions are not measured at the reconnection site, but slightly later, when VEX has already moved away, and the opposite direction can be created by the mirroring of the ions near the planet.

Acknowledgements. The authors would like to thank Rumi Nakamura for very useful discussions. The work by ZV was supported by the Austrian "Fonds zur Förderung der wissenschaftlichen Forschung" under project P20131-N16.

Editor in Chief W. Kofman thanks two anonymous referees for their help in evaluating this paper.

\section{References}

Baker, D. N., Pulkkinen, T. I., Angelopoulos, V., Baumjohann, W., and McPherron, R. L.: Neutral line model of substorms: Past results and present view, J. Geophys. Res., 101, 12975-13010, 1996.

Barabash, S., Sauvaud, J.-A., Gunell, H., et al.: The analyser of space plasmas and energetic atoms (ASPERA-4) for the Venus Express mission, Planet. Space Sci., 55, 1772-1792, doi:10. 1016/j.pss.2007.01.014, 2007.

Barabash, S., Fedorov, A., Sauvaud, J.-A., et al.: The loss of ions from Venus through the plasma wake, Nature, 450, Nature06434, doi:10.1038/nature06434, 2007. 
Baumjohann, W., Paschmann, G., and Lühr, H.: Characteristics of high-speed ion flows in the plasma sheet, J. Geophys. Res., 95, 3801-3810, 1990.

Baumjohann, W., Hesse, M., Kokubun, S., Mukai, T., Nagai, T., and Petrukovich, A. A.: Substorm dipolarization and recovery, J. Geophys. Res., 104, 24995-25000, 1999.

Baumjohann, W., Roux, A., Le Contel, O., Nakamura, R., Birn, J., Hoshino, M., Lui, A. T. Y., Owen, C. J., Sauvaud, J.-A., Vaivads, A., Fontaine, D., and Runov, A.: Dynamics of thin current sheets: Cluster observations, Ann. Geophys., 25, 1365-1389, 2007, http://www.ann-geophys.net/25/1365/2007/.

Bertucci, C., Achilleos, N., Dougherty, M. K., Modolo, R., Coates, A. J., Szego, K., Masters, A., Ma, Y., Neubauer, F. M., Garnier, P., Wahlund, J.-E., and Young, D. T.: The magnetic memory of Titan's ionized atmosphere, Science, 321, 1475-1478, doi:10. 1126/science.1159780, 2008.

Brace, L. H. and Kliore, A. J.: The structure of the Venus ionosphere, Space Sci. Rev., 55, 81-163, 1991.

Eastwood, J. P., Brain, D. A., Halekas, J. S., j. F. Drake, Phan, T. D., Øieroset, M., d. L. Mitchell, Lin, R. P., and Acuña, M.: Evidence for collisionless magnetic reconnection at Mars, Geophys. Res. Lett., 35, L02106, doi:10.1029/2007GL032289, 2008.

Fedorov, A., Ferrier, C., Sauvaud, J.-A., et al.: Comparative analysis of Venus and Mars magnetotails, Planet. Space Sci., 56, 812817, doi:10.1016/j.pss.2007.12.012, 2008.

Fujimoto, M., Baumjohann, W., Kabin, K., Nakamura, R., Slavin, J. A., Terada, N., and Zelenyi, L.: Hermean magnetosphere-solar wind interaction, Space Sci. Rev., 132, 529-550, doi:10.1007/ s11214-007-9245-8, 2007.

Ge, Y. S., Jian, L. K., and Russell, C. T.: Growth phase of Jovian substorms, Geophys. Res. Lett., 34, L23106, doi:10.1029/ 2007GL031987, 2007.

Haerendel, G.: Disruption, ballooning or auroral avalanche - On the cause of substorms, in: Proc. of the International Conference on Substorms (ICS 1), edited by: Hultqvist, B., pp. 417-420, ESA, Noordwijk, 1992.

Henderson, P. D., Owen, C. J., Alexeev, I. V., Slavin, J., Fazakerley, A. N., Lucek, E., and Rème, H.: Cluster observations of flux rope structures in the near-tail, Ann. Geophys., 24, 651-666, 2006, http://www.ann-geophys.net/24/651/2006/.

Imada, S., Hoshino, M., and Mukai, T.: Average profiles of energetic and thermal electrons in the magnetotail reconnection regions, Geophys. Res. Lett., 32, L09101, doi:10.1029/ 2005GL022594, 2005.

Imada, S., Nakamura, R., Daly, P. W., Hoshino, M., Baumjohann, W., Mühlbachler, S., Balogh, A., and Rème, H.: Energetic electron acceleration in the downstream reconnection outflow region, J. Geophys. Res., 112, A03202, doi:10.1029/2006JA011847, 2007.

Jackman, C. M., Russell, C. T., Southwood, D. J., Arridge, C. S., Achilleos, N., and Dougherty, M. K.: Strong rapid dipolarizations in Saturn's magnetotail: In situe evidence of reconnection, Geophys. Res. Lett., 34, L11203, doi:10.1029/2007GL029764, 2007.

Kallio, E., Zhang, T. L., Barabash, S., et al.: The Venusian induced magnetosphere: A case study of plasma and magnetic field measurements on the Venus Express mission, Planet. Space Sci., 56, 769-801, doi:10.1016/j.pss.2007.09.011, 2008.

Kronberg, E. A., Woch, J., Krupp, N., Lagg, A., Khurana, K. K., and Glassmeier, K.-H.: Mass release at Jupiter: Substorm-like processes in the Jovian magnetotail, J. Geophys. Res., 110, A03211, doi:10.1029/2004JA010777, 2005.

Kronberg, E. A., Woch, J., Krupp, N., Lagg, A., Daly, P. W., and Korth, A.: Comparison of periodic substorms at Jupiter and Earth, J. Geophys. Res., 113, A04212, doi:10.1029/ 2007JA012880, 2008.

Luhmann, J. G.: The solar wind interaction with Venus, Space Sci. Rev., 44, 241-306, 1986.

Luhmann, J. G., Brecht, S. H., Spreiter, J. R., Stahara, S. S., Steinolfson, R. S., and Nagy, A. F.: Global models of the solar wind interaction with Venus, in: Venus II, edited by: Bougher, S. W., Hunten, D. M., and Philips, R. J., pp. 33-60, University of Arizona Press, Tucson, 1997.

Lui, A. T. Y.: Electric current approach to magnetospheric physics and the distinction between current disruption and magnetic reconnection, in: Magnetospheric Current Systems, edited by: Ohtani, S.-I., Fujii, R., Hesse, M., and Lysak, R. L., pp. 31-40, AGU, Washington, 2000.

Mitchell, D. G., Brandt, P. C., Roelof, E. C., Dandouras, J., Krimigis, S. M., Mauk, B. H., Paranicas, C. P., Krupp, N., Hamilton, D. C., Kurth, W. S., Zarka, P., Dougherty, M. K., Bunce, J. E., and Shemansky, D. E.: Energetic ion acceleration in Saturn's magnetotail: Substorms at Saturn?, Geophys. Res. Lett., 32, L20S01, doi:10.1029/2005GL022647, 2005.

Murabashi, K., Grebowsky, J. M., Taylor Jr., H. A., Luhmann, J. G., Russell, C. T., and Barnes, A.: Magnetic field in the wake of Venus and the formation of ionospheric holes, J. Geophys. Res., 90, 1385-1398, 1985.

Parker, E. N.: The alternative paradigm for magnetospheric physics, J. Geophys. Res., 101, 10587-10625, 1996.

Pérez-de-Tejada, H.: Distribution of plasma and magnetic fluxes in the Venus near wake, J. Geophys. Res., 91, 8039-8044, 1986.

Pérez-de-Tejada, H., Dryer, M., Intrilligator, D. S., Russell, C. T., and Brace, L. H.: Plasma distribution and magnetic field orientation in the Venus near wake: Solar wind control of the nightside ionopause, J. Geophys. Res., 88, 9019-9025, 1983.

Petrukovich, A. A., Baumjohann, W., Nakamura, R., Runov, A. V., and Balogh, A.: Cluster vision of the magnetotail current sheet on a macroscale, J. Geophys. Res, 110, A06204, doi:10.1029/2004JA010825, 2005.

Phillips, J. L. and McComas, D. L.: The magnetosheath and magnetotail of Venus, Space Sci. Rev., 55, 1-80, 1991.

Runov, A., Nakamura, R., Baumjohann, W., Treumann, R. A., Zhang, T. L., Volwerk, M., Vörös, Z., Balogh, A., Glassmeier, K.-H., Klecker, B., Rème, H., and Kistler, L.: Current sheet structure near magnetic X-line observed by Cluster, Geophys. Res. Lett., 30, 1579, doi:10.1029/2002GL016730, 2003.

Runov, A., Sergeev, V. A., Nakamura, R., Baumjohann, W., Zhang, T. L., Asano, Y., Volwerk, M., Vörös, Z., Balogh, A., and Rème, H.: Reconstruction of the magnetotail current sheet structure using multi-point Cluster measurements, Planet. Space Sci., 53, 237-243, 2005.

Russell, C. T., Luhmann, J. G., Elphic, R. C., and Scarf, F. L.: The distant bow shock and magnetotail of Venus: Magnetic field and plasma wave observations, Geophys. Res. Lett., 8, 843-846, 1981.

Russell, C. T., Saunders, M. A., Phillips, J. L., and Fedder, J. A.: Near-tail reconnection as the cause of cometary tail disconnec- 
tions, J. Geophys. Res., 91, 1417-1423, 1986.

Russell, C. T., Jackman, C. M., Wei, Y. H., Bertucci, C., and Dougherty, M. K.: Titan's influence on Saturnian substorm occurrence, Geophys. Res. Lett., 35, L12105, doi:10.1029/ 2008GL034080, 2008.

Saunders, M. A. and Russell, C. T.: Average dimension and magentic structure of the distant Venus magnetotail, J. Geophys. Res., 91, 5589-5604, 1986.

Sergeev, V., Runov, A., Baumjohann, W., Nakamura, R., Zhang, T. L., Volwerk, M., Balogh, A., Rème, H., Sauvaud, J.-A., André, M., and Klecker, B.: Current sheet flapping motion and structure observed by Cluster, Geophys. Res. Lett., 30, 1327, doi:10.1029/ 2002GL016500, 2003.

Sonnerup, B. U. Ö.: Magnetic field reconnection, in: Solar System Plasma Physics, vol. III, edited by: Lanzerotti, L. T., Kennel, C. F., and Parker, E. N., pp. 45-108, North-Holland, New York, 1979.

Sonnerup, B. U. Ö. and Scheible, M.: Minimum and maximum variance analysis, in: Analysis Methods for Multi-Spacecraft Data, edited by: Paschmann, G. and Daly, P., pp. 185-220, ESA, Noordwijk, 1998.

Volwerk. M., Nakamura, R., Baumjohann, W., Treumann, R. A., Runov, A., Vörös, Z., Zhang, T. L., Asano, Y., Klecker, B., Richter, I., Balogh, A., and Rème, H.: A statistical study of compressional waves in the tail current sheet, J. Geophys. Res., 108, 1429, doi:10.1029/2003JA010155, 2003.

Volwerk, M., Baumjohann, W., Glassmeier, K. H., Nakamura, R., Zhang, T. L., Runov, A., Vörös, Z., Klecker, B., Treumann, R. A., Bogdanova, Y., Eichelberger, H.-U., Balogh, A., and Rème, H.: Compressional waves in the Earth's neutral sheet, Ann. Geophys., 22, 303-315, 2004, http://www.ann-geophys.net/22/303/2004/.

Volwerk, M.: Multi-Satellite observations of ULF waves, in: Magnetospheric ULF waves: Synthesis and new directions, edited by: Takahashi, K., Chi, P. J., Denton, R. E., and Lysak, R. L., pp. 109-135, AGU, Washington, 2006.
Vörös, Z., Zhang, T. L., Leubner, M. P., Volwerk, M., Delva, M., and Baumjohann, W.: Intermittent turbulence, noisy fluctuations and wave structures in the Venusian magnetosheath and wake, J. Geophys. Res., 113, E00B21, doi:10.1029/2008JE003159, 2008a.

Vörös, Z., Zhang, T. L., Leubner, M. P., Volwerk, M., Delva, M., Baumjohann, W., and Kudela, K.: Magnetic fluctuations and turbulence in the Venus magnetosheath and wake, Geophys. Res. Lett., 35, L11102, doi:10.1029/2008GL033879, 2008b.

Vourlidas, A., Davis, C. J., Eyles, C. J., Crothers, S. R., Harrison, R. A., Howard, R. A., Moses, J. D., and Socker, D. G.: First Direct Observation of the Interaction between a Comet and a Coronal Mass Ejection Leading to a Complete Plasma Tail Disconnection, Astrophys. J., 668, L79-L82, doi:10.1086/522587, 2007.

Zhang, T. L., Baumjohann, W., Delva, M., et al.: Magnetic field investigation of the Venus plasma environment: expected new results, Planet. Space Sci., 54, 1336-1343, 2006.

Zhang, T. L., Delva, M., Baumjohann, W., et al.: Little or no solar wind enters Venus atmosphere at solar minimum, Nature, 450, 654-656, doi:10.1038/nature06026, 2007.

Zhang, T. L., Delva, M., Baumjohann, W., Volwerk, M., Russell, C. T., Barabash, S., Balikhin, M., Pope, S., Glassmeier, K.-H., Wang, C., and Kudela, K.: Initial venus express magnetic field observations of the magnetic barrier at solar minimum, Planet. Space Sci., 56, 790-795, doi:10.1016/j.pss.2007.10.013, 2008a.

Zhang, T. L., Delva, M., Baumjohann, W., Volwerk, M., Russell, C. T., Barabash, S., Balikhin, M., Pope, S., Glassmeier, K.-H., Kudela, K., C.Wang, Vörös, Z., and Zambelli, W.: Initial Venus express magnetic field observations of the Venus bow shock location at solar minimum, Planet. Space Sci., 56, 785-789, doi: 10.1016/j.pss.2007.09.012, 2008b. 